\title{
Whole-Body Pregnant Woman Modeling By Digital Geometry Processing With Detailed Uterofetal Unit Based on Medical Images
}

\author{
Lazar Bibin, Jérémie Anquez, Juan Pablo de la Plata Alcalde, Tamy Boubekeur, \\ Elsa D. Angelini, Member, IEEE, and Isabelle Bloch*, Member, IEEE
}

\begin{abstract}
Anatomical models of pregnant women are used in several applications, such as numerical dosimetry, to assess the potential effects of electromagnetic fields on biological tissues, or medical simulation. Recent advances in medical imaging have enabled the generation of realistic and detailed models of human beings. The construction of pregnant woman models remains a complex task, since it is not possible to acquire whole-body images. Only few models have been developed up to now, and they all present some limitations regarding the representation of anatomical variability of the fetus shape and position over the entire gestation. This paper describes a complete methodology that intends to automate each step of the construction of pregnant women models. The proposed approach relies on the segmentation of 3-D ultrasonic and 3-D magnetic resonance imaging (MRI) data, and on dedicated computer graphics tools. The lack of complete anatomical information for the mother in image data is compensated, in an original way, by merging the available information with a synthetic woman model, deformed to match the image-based information. A set of models anatomically validated by clinical experts is presented. They include detailed information on uterofetal units and cover different gestational stages with various fetal positions.
\end{abstract}

Index Terms-Anatomical modeling, computer graphics, fetus, medical imaging, mesh generation and deformation, MRI, pregnant woman, segmentation, three-dimensional ultrasound (3-DUS).

\section{INTRODUCTION}

$\mathbf{N}$ UMERICAL dosimetry focuses on the computation of absorbed dose by body tissues from exposure to ionizing and nonionizing radiation. Such studies require realistic models of the human body, for example, to monitor the absorption of electromagnetic energy in biological tissues emitted by mobile phones. With the advent of fast whole-body acquisition imaging protocols, voxel-based models can, nowadays, be built using

Manuscript received January 9, 2010; revised April 23, 2010; accepted May 9, 2010. Date of publication June 21, 2010; date of current version September 15, 2010. This work was supported by the Instituts Carnot-Institut Telecom, Orange Laboratories R\&D, and Fondation Santé et Radiofréquences. Asterisk indicates corresponding author.

L. Bibin, J. Anquez, J. P. de la Plata Alcalde, T. Boubekeur, and E. D. Angelini are with the Institut Telecom; Telecom ParisTech; CNRS LTCI; WHIST Lab, Paris, France (e-mail: lazarbibin@hotmail.com; jeremie.anquez@telecom-paristech.fr; delaplat@telecom-paristech.fr; tamy. boubekeur@telecom-paristech.fr; elsa.angelini@telecom-paristech.fr).

${ }^{*}$ I. Bloch is with the Laboratoire Traitement et Communication de 1'Information, Wave Human Interactions and Telecommunications Laboratory, Institut Télécom, Télécom ParisTech, National Scientific Research Center, Paris 75634, France (e-mail: isabelle.bloch@enst.fr).

Color versions of one or more of the figures in this paper are available online at http://ieeexplore.ieee.org.

Digital Object Identifier 10.1109/TBME.2010.2053367 segmented medical data acquired from volunteers. Numerous adult and children voxel-based models are now available [1], [2], which have enabled extensive dosimetry studies. In 2006, the World Health Organization ${ }^{1}$ designated studies aiming at assessing fetal exposure during pregnancy as a new priority.

Since whole-body medical imaging data cannot be acquired from pregnant women for ethical reasons (related to the fetus exposure) and technical limitations (related to the scanning time), hybrid models can only be built by merging stylized models (with organs represented by surface equations), voxel-based models and/or synthetic models from the computer graphics community. In addition, only few works have proposed models of pregnant women at different stages of pregnancy.

In this paper, we describe a complete methodology to build whole-body pregnant woman models, embedding detailed and realistic uterofetal unit (UFU) models at various stages of gestation and in different positions. This paper intends to complement the set of existing models by providing more automated computational tools.

Regarding the UFU modeling part, realism is ensured by the exploitation of medical images, obtained with two modalities used in pregnancy follow up: 3-D ultrasound (3-DUS) during the first trimester and magnetic resonance imaging (MRI) during the second and third trimesters.

Detailed UFU segmentations, validated by expert clinicians, are exploited by several digital geometry tools. Organ contours are extracted from medical images and represented as smooth meshed surfaces to enable fine rasterization, i.e., discretization of the shapes on a Cartesian grid. Surface smoothing prevents "staircase" effects that can be observed when a voxel model is sampled at a low resolution with a naive method (e.g., nearest neighbor interpolation). These singularities are undesirable as they induce some bias in the dosimetry simulations.

We propose an automated framework for the insertion and placement of the UFU into a whole-body woman model, which is usually done manually. Automating this task enables the generation of multiple models, while limiting manual and subjective interactions. We have selected an homogeneous graphical whole-body woman model called Victoria provided by Daz Studio (www.daz3d.com), which presents the advantages of being easily deformable and can be positioned in different postures.

\footnotetext{
${ }^{1}$ http://www.who.int/peh-emf/research/rf_research_agenda_2006.pdf
} 


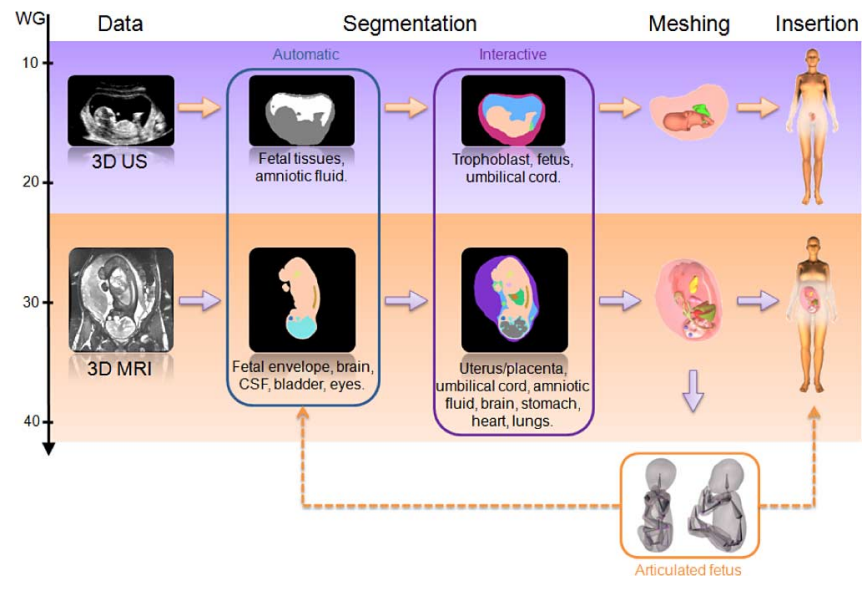

Fig. 1. Overview of the proposed methodology for whole-body pregnant woman modeling. WG stands for weeks of gestation.

After reviewing the state-of-the-art in terms of pregnant woman modeling, we describe the medical image database used in this paper. Then, we present the computational framework exploited to generate realistic models with detailed UFU, which are automatically inserted into a synthetic woman body that is enlarged to host the whole uterus. Finally, to guarantee the representation of the major variations in anatomical configurations and morphologies, we have designed a tool to control the fat distribution on the pregnant woman body envelope and to modify the UFU position between standing and laying positions of the woman. We focused on this tissue because of its importance in numerical dosimetry. Visual illustrations of some models generated in this study are provided and discussed.

Fig. 1 illustrates the proposed scheme for whole-body pregnant woman modeling. From a 3-DUS database for the first trimester and a 3-D MRI database for the second and third trimesters, a segmentation of different visible tissues is performed using automated or interactive tools (see Section III). Next, 3-D surface meshes of the UFUs are generated (see Section IV), and an articulation of the fetal envelope is proposed to enable arbitrary positioning of the fetus inside the uterus (see Section VI). Final insertion of the UFU inside a generic nonpregnant woman body envelope is performed automatically (see Section V).

\section{OVERVIEW of Existing Pregnant Woman Models}

Existing pregnant woman models used for dosimetry can be categorized into four classes, depending on the type of representation: 1) mathematical models, where anatomical structures are described using surface equations; 2) voxelized models based on medical images; 3 ) graphical models synthesized by the computer graphics community; and 4) hybrid models combining mathematical, voxelized, or synthetic models. The mathematical (stylized) models were considered in pioneer works for their ease of implementation, but are inherently limited in terms of anatomical realism. Voxelized models, built with segmented medical images, are now preferred as they represent accurately the human anatomy. However, medical image data is not always available and its segmentation requires an important amount of manual interaction. This has motivated the introduction of graphical (synthetic) models, built with computer graphics softwares, such as in [10]. Most satisfying models were generated under supervision of experienced anatomists, to ensure their realism.

The first published pregnant woman models were mathematical models used in [3], which were obtained by inserting a mathematical shape representing the fetus into a nonpregnant mathematical woman model [11]. The shape and the position of the female organs were modified to insert the fetus, which was rotated by $33.1^{\circ}$ from the horizontal plane for a model of the first trimester of gestation and by $40^{\circ}$ for models of the last two trimesters. Three pregnant women at 3,6, and 9 months of gestation were represented. The use of mathematical shapes enabled fast computation and easy manipulation of the models, but severely affected their realism and usability.

In [12], a voxelized model was built from a computerized tomography (CT) dataset acquired from the torso of corpulent pregnant woman. The fetus model was rather coarse due to the large image slice thickness $(7 \mathrm{~mm})$, and only fetal soft tissues and skeleton were distinguished. The segmentation of these structures was manually performed by the authors with the help of anatomical atlases. In [13], segmentations of the maternal trunk, the uterus and the gestational sac (when visible) from CT data were used to generate UFUs between the 12th and the 36th week of gestation (WG), distinguishing soft tissues from bones. However, whole-body models, i.e., representing the body from head to toe, are desirable to study the influence of plane waves emitted by, for example, phone masts. The few works dedicated to model the entire pregnant woman at different stages of pregnancy were based on hybrid modeling, which relies on the combination of mathematical, voxelized, and/or synthetic models.

The hybrid model SILVY, presented in [4], combined a malformed fetus segmented from a MRI dataset, the voxelized pregnant woman trunk described in [12] and the homogeneous whole-body envelope of a woman generated by laser scan imaging. Nonlinear scaling was used to adapt the whole-body envelope to the trunk model after removing the superficial fat and skin layers. The brain and spinal cord of the model NORMAN [14] were also inserted and fitted into SILVY.

In [5], a set of mathematical models of the uterus and fetus at $8,13,26$, and 38 WG [15] were voxelized and embedded in the nonpregnant voxel model NAOMI [16]. Voxel editing was required to translate and deform NAOMI organs away from the uterus.

In [6], nine pregnant female models at each month of gestation were generated, embedding a UFU (which included the placenta), a bladder, and bones (based on segmented MRI data), into a computer graphics woman model. The abdomen of the woman was only scaled for models at stages beyond 4 months and the UFUs were scaled to simulate the different gestational ages.

In [7], hybrid models using the UFU and maternal organs models from [12], the VIP-MAN model from [17], and computer graphics models were used together to construct 3-D surface 
TABLE I

Comparative Table of Existing Pregnant Woman Models

\begin{tabular}{|c|c|c|c|c|c|c|}
\hline & $\begin{array}{c}\text { mother model } \\
\text { type }\end{array}$ & $\begin{array}{l}\text { UFU model } \\
\text { type }\end{array}$ & $\begin{array}{c}\text { \# of maternal } \\
\text { tissues }\end{array}$ & $\begin{array}{c}\# \text { of UFU } \\
\text { tissues }\end{array}$ & $\begin{array}{c}\text { Pregnancy stages } \\
\text { (WG) }\end{array}$ & $\begin{array}{c}\text { Fetal position } \\
\text { variability }\end{array}$ \\
\hline M.G. Stabin [3] & mathematical & mathematical & 32 & 5 & $13,26,38$ & no \\
\hline SILVY [4] & graph./voxel. & voxelized & 29 & 3 & 30 & no \\
\hline P.J. Dimbylow [5] & voxelized & mathematical & 41 & 5 & $8,13,26,38$ & no \\
\hline D. Wu [6] & graphical & voxelized & 3 & 3 & $4,8,13,18,22,26,30,34,38$ & no \\
\hline X.G. Xu [7] & graph./voxel. & graph./voxel. & 29 & 3 & $13,26,38$ & no \\
\hline T. Nagaoka [8] & voxelized & voxelized & 51 & 6 & 26 & no \\
\hline Katja [9] & voxelized & voxelized & 153 & 18 & 24 & no \\
\hline Our models & graphical & voxelized & 2 & 4-7 (3DUS), 11 (MRI) & $6,7,8,11,24,28,30,31,33$ & yes \\
\hline
\end{tabular}

Pregnancy stages are expressed in WG.

models of a pregnant woman with detailed organs. Three models were built at 3,6, and 9 months of pregnancy. To insert the fetus, the maternal organs were manually translated and deformed to avoid overlaps using free-form deformation (FFD) lattices. An angle of $50^{\circ}$ was applied between the caudocephalic axis of the female body and the fetus for the 6 and 9 months models, and $60^{\circ}$ for the 3 months model. Fetal soft tissues, brain, and skeleton were distinguished.

In [8], a voxelized model of the UFU at 26 WG was extracted from MRI data (with the fetal brain, fetal eyes, amniotic fluid, placenta, and uterine wall). By scaling down this UFU model, two other models were generated corresponding to 13 and 18 WG. Using FFDs, the models were embedded inside a detailed voxelized nonpregnant woman model [18], exploiting information from the MRI of the pregnant woman abdomen. The position fetus model was in left occiput anterior position with respect to the mother's pelvis, i.e., the fetal occiput was oriented toward the mother's left anterior side.

Katja, proposed in [9], is a pregnant woman model combining a detailed UFU modeled from MRI data and the voxelized female model International Committee of Radiological Protection (ICPR)-AF. The insertion of the UFU into the voxelized nonpregnant model required voxel editing, performed with a dedicated software [19].

Table I compares the most sophisticated existing pregnant woman models according to the type of models, the number of represented tissues, the coverage of pregnancy stages, and the variability of the fetal position.

In this paper, we propose to contribute to the field of whole pregnant woman modeling by presenting a complete methodology to create several hybrid models with a better coverage of the whole period of gestation, and higher levels of anatomical details on the UFU. Our models include detailed UFUs, based on segmentation results from 3-DUS and MRI data, with various fetal development stages and positions. We decided to represent part of the anatomical variability of fetal development and fetus positioning within the uterus by using a large database of fetal images (i.e., a large set of observations), which were segmented to extract the useful information for modeling purpose, i.e., the UFU organs. We, therefore, chose not to build on existing pregnant women models, but rather construct a novel methodology exploiting state-of-the-art medical imaging data from two routine screening modalities. This approach has enabled us to collaborate with a large team of clinical experts, to control the anatomical accuracy of our fetal models. We believe that this is a radically different approach from what was performed in the existing works reviewed. Some original features of our modeling framework include the automation of some segmentation tasks and the automation of the UFU insertion process.

\section{UFU SEGMENTATION}

To create realistic models of the UFU, we used medical images acquired with 3-DUS and MRI. These two types of medical imaging modalities allow us to cover the three trimesters of pregnancy. Indeed, during the first trimester, only US examinations are allowed and the field of view of the US probes can include the whole UFU until around the 16th WG. While MRI screening is not performed during the first trimester to limit the fetus exposure to electromagnetic fields, high-quality 3-D volumes can be acquired during the second and third trimesters, and used to generate realistic UFU models from segmentation results.

\section{A. Segmentation of 3-DUS Data}

1) 3-DUS Database: With the collaboration of obstetricians from Port-Royal (Paris, France) and Beaujon hospitals (Clichy, France), we obtained 18 3-DUS sequences between 6 and 11 WG with high-image quality, acquired on a VOLUSON 730 machine (General Electric, Milwauke, WI). These images have submillimetric isotropic resolution (typically $0.6 \times 0.6 \times 0.6 \mathrm{~mm}^{3}$ ). The whole embryo and all the important maternal uterine tissues are visible on these images. While the 3-DUS images used in this paper have a high-spatial resolution, the overall image quality is limited. Nevertheless, this is the imaging modality of choice, worldwide, for monitoring fetal development over the entire pregnancy. Obstetricians perform measurements on these images and a large list of clinical papers have confirmed the accuracy of these measures to correctly assess fetal development and even fetal body weight.

2) Segmentation: To segment the 3-DUS images, we first classify the voxels into two classes: the amniotic fluid on the one hand, and the embryofetal and maternal tissues, on the other hand. This is performed using the method described in [20], where statistical distributions of intensities within each class are integrated in a deformable model. The classification results are postprocessed manually to disconnect the embryofetal body from the uterine wall and from the umbilical cord using the opensource softwares MIPAV (http://mipav.cit.nih.gov/) and Slicer (http://www.slicer.org/). Finally, additional tissues are manually identified. The following uterofetal structures are systematically 

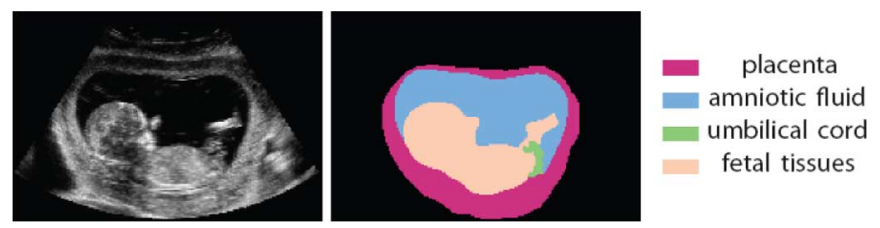

Fig. 2. Slice of a 3-DUS volume of a fetus aged of $11 \mathrm{WG}$ and its corresponding segmentation result.

segmented on the US images: the fetus, the amniotic fluid, the umbilical cord, and the placenta. Depending on the fetus gestational age and on the width of the field of view exploited during the acquisition, we can also identify: the yolk sac, the endometrium, and the myometrium. The volumes of interest of the different structures were validated by an expert obstetrician and converted into labeled masks. Fig. 2 shows one slice of a 3-DUS volume of a fetus aged of $11 \mathrm{WG}$ and its corresponding segmentation result.

Given the recent introduction of 3-DUS systems for obstetric screening and the constant improvement in US imaging quality, our methodological work on the segmentation of this type of data should contribute to the development of more precise anatomical modeling and quantification from 3-DUS data. All the segmentations performed on 3-DUS data were considered precise enough by the medical experts to be used in our models.

\section{B. Segmentation of MRI Data}

1) MRI Database: In collaboration with pediatric radiologists from the Cochin-Saint Vincent de Paul hospital (Paris, France), a study [21] was performed to select the best-suited MRI imaging protocols for the segmentation of the fetus and the maternal body envelope. Images were acquired using the generic sequence steady-state free precession (SSFP). This sequence enables to acquire image volumes encompassing the UFU in less than $30 \mathrm{~s}$. Thus, images free from fetal-motionrelated artifacts can be obtained, guaranteeing 3-D consistency of the UFU anatomical structures. Moreover, intensities in fluids are strikingly higher than in fetal soft tissues and high contrasts enable easy delineation of the fetus and its anatomy. Image quality criteria include the following: large field of view to image the whole uterus, good global contrast, good spatial resolution (typically $1 \times 1 \times 4 \mathrm{~mm}^{3}$ ), fast acquisition (less than $30 \mathrm{~s}$ ), and low sensitivity to fetal movements artifacts. The SSFP sequence was chosen as the one best satisfying these criteria. The database gathered for this paper includes 43 cases between 24 and 33 WG. During the second trimester, the gestational sac volume, containing the amniotic fluid, is large compared to the fetus size. Hence, fetal motion artifacts are frequently observed in MRI images, preventing accurate segmentation of the fetus. Few data were therefore gathered during this period, while most data were acquired during the third trimester, when fetal motion is limited.

2) Segmentation: On MRI data, we segmented the maternal tissues, uterine wall/placenta, amniotic fluid, umbilical cord, and the fetus body envelope using semiautomated segmentation tools. Inside the fetus body, the fetal anatomy was detailed by

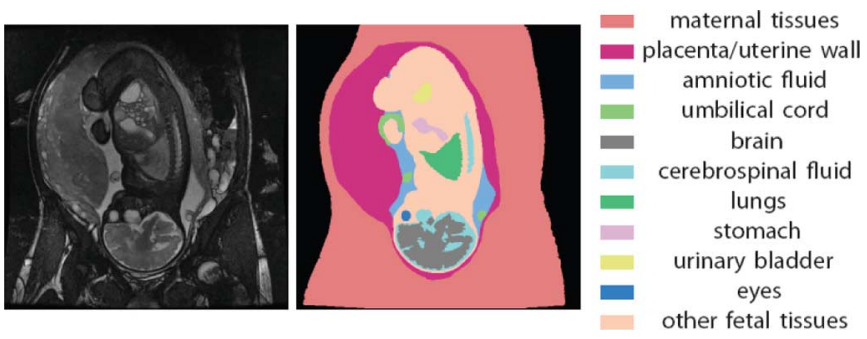

Fig. 3. MRI slice of a 3-D volume of a fetus of $33 \mathrm{WG}$ and its corresponding segmentation results.

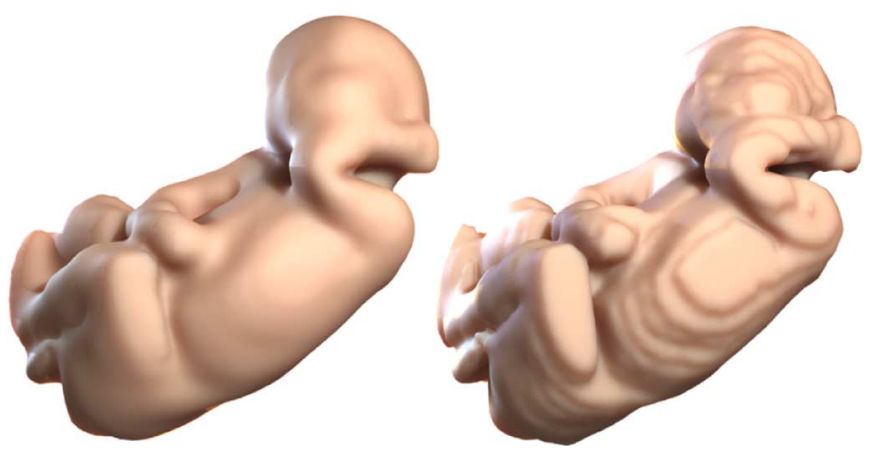

Fig. 4. Illustration on a fetus body of the (left) proposed surface reconstruction versus (right) direct meshing from image segmentation.

segmenting the brain, cerebrospinal fluid, spine, eyes, lungs, heart, stomach, and urinary bladder. The overall segmentation process is time consuming and automated approaches were developed for certain organs. In particular, a novel automated segmentation method based on morphological information and deformable models [22] provided very accurate results for the eyes, brain, and cerebrospinal fluid. A graph-cut approach was also proposed to automatically segment the fetal body envelope initialized with a generic articulated fetus model described in Section VI [23]. A MRI slice from a 3-D volume of a fetus at $33 \mathrm{WG}$ is displayed in Fig. 3, along with the corresponding segmentation results, validated by an obstetrician.

\section{3-D Surface Mesh Modeling}

For nonionizing dosimetry studies, the finite-difference timedomain method [24] is frequently used for numerical simulations on a spatial grid, with labeled anthropomorphic models, which need to be smooth to avoid simulation bias on singularities. Naive direct meshing approaches generate "staircase" effects (see Fig. 4) on the surface models, especially when MRI images with anisotropic resolution are considered. In this section, we describe a method for generating a high-quality triangle mesh from a presegmented volume of interest [25]. We adopt a generic approach by first extracting an unorganized set of points from the volume data, and then, generating a mesh by sampling a smooth surface, which approximates this point set. The entire process is performed in a few seconds.

The mesh reconstruction algorithm is composed of three main steps, detailed as following and illustrated in Fig. 5. With this algorithm, a mesh of arbitrary resolution can be extracted from 


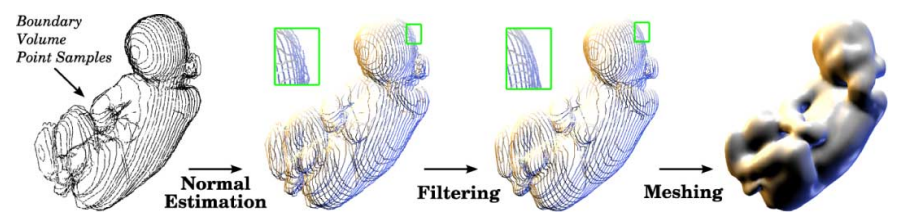

Fig. 5. Illustration on a fetus body of the reconstruction pipeline.

an arbitrary sparse point set. Consequently, the system allows producing dense enough surface sampling to enable both accurate subsequent deformations of the objects and robust rasterization within the original volume domain.

\section{A. Point-Normal Sampling}

In addition to the positions $\mathbf{p}_{i}$ of point samples, the algorithm requires surface normal estimates $\mathbf{n}_{i}$ at these points, in order to generate a mesh $\mathcal{M}$ from the point-normal sampling $\mathcal{P}^{N}=\left\{\left\{\mathbf{p}_{0}, \mathbf{n}_{0}\right\}, \ldots\left\{\mathbf{p}_{m}, \mathbf{n}_{m}\right\}\right\}$. We compute an approximate normal vector at each point by using a principal component analysis [26] in 3-D space. More precisely, the normal is given as $\mathbf{u}_{i}$, the eigenvector associated with the smallest eigenvalue of the covariance matrix of the $k$-nearest neighborhood of $\mathbf{p}_{i}$. In practice, the anisotropic nature of the original sampling induces a rather large value for $k$, usually $k \in[50,120]$ (the exact value can be tuned according to the image resolution and to the desired level of smoothness), and we use a kD-tree data structure [27] to efficiently compute the $k$-neighborhood queries.

In our case, the sign of the normal can be resolved using additional information available from the volume data: the vector $\mathbf{a}_{i}$, from the surface point $\mathbf{p}_{i}$ to the corresponding point on a dilated volume boundary, always points from the surface to the outside of the object. Thus, we get

$$
\mathbf{n}_{i}=\frac{\overline{\mathbf{n}_{i}}}{\left\|\overline{\mathbf{n}_{i}}\right\|} \text { with } \overline{\mathbf{n}_{i}}= \begin{cases}\mathbf{u}_{i}, & \text { if } \mathbf{u}_{i} \cdot \mathbf{a}_{i}>0 \\ -\mathbf{u}_{i}, & \text { otherwise }\end{cases}
$$

Note that $\mathbf{n}_{i}$ is only an estimate, with a smoothness controlled by $k$. To increase the quality of this estimate for later stages of the reconstruction pipeline, the normals are recomputed after each major processing step in this algorithm.

\section{B. Moving Least-Square Projection Operator}

The moving least-square (or MLS) projection operator [28], [29] is a scattered data approximation method intensively used in geometry processing. We make use of it for both filtering and meshing steps, as it provides a simple and powerful tool for the sparse sampling $\mathcal{P}^{N}$ extracted from volume data. We consider a variation of a recent simplification of the MLS operator [30]. Let consider $\mathbf{q} \in \mathbb{R}^{3}$. The MLS operator is defined as follows:

$$
\operatorname{MLS}^{\mathcal{P}^{N}}: \mathbb{R}^{3} \rightarrow \mathbb{R}^{3}, \mathbf{q} \rightarrow \Pi^{\infty}(\mathbf{q})
$$

It is based on the orthogonal projection $\Pi(\mathbf{q})$ of $\mathbf{q}$ onto a weighted least-square plane $H_{\mathbf{q}}=\{c(\mathbf{q}), n(\mathbf{q})\}$

$$
\Pi(\mathbf{q})=\mathbf{q}-<(\mathbf{q}-c(\mathbf{q})) \cdot n(\mathbf{q})>n(\mathbf{q})
$$

where

$$
c(\mathbf{q})=\frac{\sum_{i} \omega\left(\left\|\mathbf{q}-\mathbf{p}_{\mathbf{i}}\right\|\right) \mathbf{p}_{\mathbf{i}}}{\sum_{i} \omega\left(\left\|\mathbf{q}-\mathbf{p}_{\mathbf{i}}\right\|\right)}, n(\mathbf{q})=\frac{\sum_{i} \omega\left(\left\|\mathbf{q}-\mathbf{p}_{\mathbf{i}}\right\|\right) \mathbf{n}_{\mathbf{i}}}{\left\|\sum_{i} \omega\left(\left\|\mathbf{q}-\mathbf{p}_{\mathbf{i}}\right\|\right) \mathbf{n}_{\mathbf{i}}\right\|}
$$

define a weighted combination over a local neighborhood of surface point samples near $\mathbf{q}$ with $\left\{\mathbf{p}_{i}, \mathbf{n}_{i}\right\} \in \mathcal{P}^{N}$. For efficiency reasons, we use Wendland's [31] compactly supported, piecewise polynomial function as the weighting kernel

$$
\omega(t)= \begin{cases}\left(1-\frac{t}{h}\right)^{4}\left(\frac{4 t}{h}+1\right), & \text { if } 0 \leq t \leq h \\ 0, & \text { if } t>h\end{cases}
$$

where $h$ controls the size of the support (i.e., smoothness). By applying iteratively this projection procedure, we define $\Pi^{j+1}(\mathbf{q})=\Pi\left(\Pi^{j}(\mathbf{q})\right)$ and generate a sequence of points $\left\{\mathbf{q}, \Pi(\mathbf{q}), \ldots, \Pi^{j}(\mathbf{q}), \ldots\right\}$ which-considering $\mathbf{q}$ in the vicinity of $\mathcal{P}^{N}$ [32] - converges toward a stationary point $\Pi^{\infty}(\mathbf{q})$. The set of points in $\mathbb{R}^{3}$, which are stationary by this MLS projection of $\mathcal{P}^{N}$ is called the point set surface (PSS) [33] of $\mathcal{P}^{N}$. This procedure converges very quickly in the vicinity of $\mathcal{P}^{N}$. In practice, we bound the number of iterations to five and the precision to a user-defined value, i.e., $\left\|\Pi^{j+1}(\mathbf{q})-\Pi^{j}(\mathbf{q})\right\|<\epsilon$. Note that $\omega(t)$ has a compact support, which allows us to consider only a small and local set of neighbors in $\mathcal{P}^{N}$. Again, a $\mathrm{kD}$-tree is used to query them in logarithmic time.

Finally, although the size of the weighting kernel allows us to control the low-pass filtering effect, this operator may notably "shrink" the shape. In such cases, we switch to an alternative evaluation procedure, designed for Hermite PSS [34]. This operator is very similar to the standard one, but better approximates Hermite data, which naturally leads to better volume preservation. In practice, the only difference resides in the way we compute the weighted center of $H_{\mathbf{q}}$ at each step

$$
c(\mathbf{q})=\frac{\sum_{i} \omega\left(\left\|\mathbf{q}-\mathbf{p}_{\mathbf{i}}\right\|\right) h_{i}(\mathbf{q})}{\sum_{i} \omega\left(\left\|\mathbf{q}-\mathbf{p}_{\mathbf{i}}\right\|\right)}
$$

where the projection of $\mathbf{q}$ onto the tangent space of $\mathbf{p}_{\mathbf{i}}$ is interpolated instead of $\mathbf{p}_{\mathbf{i}}$ itself

$$
h_{i}(\mathbf{q})=\mathbf{q}-<\left(\mathbf{q}-\mathbf{p}_{\mathbf{i}}\right) \cdot \mathbf{n}_{\mathbf{i}}>\mathbf{n}_{\mathbf{i}} .
$$

As mentioned by Alexa and Adamson [34], it is straightforward to interpolate between standard and Hermite evaluation, offering an additional intuitive control parameter.

\section{Filtering of Point Set $\mathcal{P}^{N}$}

Manual segmentation may often lead to inaccurate volume boundary and unwanted samples in $\mathcal{P}^{N}$. Consequently, we need to filter $\mathcal{P}^{N}$ prior to the mesh generation stage. In practice, this filtering boils down to smooth $\mathcal{P}^{N}$ and remove its outliers, which are samples located far away from the estimated surface. The former can be addressed by applying the MLS projection on every sample of $\mathcal{P}^{N}$, using $h$ to control the low-pass filtering effect (i.e., $\mathcal{P}^{N}$ is projected onto the PSS it defines). We address the later problem using an iterative classification inspired from the method of Bradley et al. [35]: we compute the plane fit criterion proposed by Weyrich et al. [36], remove the detected 
outliers and restart with a quadratically decreasing bound until a user-defined threshold. Our experiments show that the number of iterations can be fixed to three and the same value was used for all processed cases.

\section{Mesh Generation}

We finally generate the mesh $\mathcal{M}$ from $\mathcal{P}^{N}$ using two distinct approaches.

1) In most cases, we use the implicit form of the PSS

$$
f^{\mathcal{P}^{N}}: \mathbb{R}^{3} \rightarrow \mathbb{R}, \mathbf{q} \rightarrow n(\mathbf{q})^{\top}(\mathbf{q}-c(\mathbf{q}))
$$

to define a scalar field with zero set- $f^{\mathcal{P}^{N}}(\mathbf{q})=0-$ corresponding to a smooth surface approximating $\mathcal{P}^{N}$. We contour it by feeding the extended marching cube algorithm [37] with $f^{\mathcal{P}^{N}}$ (i.e., $f^{\mathcal{P}^{N}}$ is evaluated at marching cube grid vertices).

2) In some cases, exhibiting large missing surface regions in the sampling obtained from volume data, we use the Poisson reconstruction algorithm, as proposed by Kazhdan et al. [38]. We use their code, publicly available.

As a result, we obtain a triangle mesh $\mathcal{M}$ sampling at arbitrary precision (controlled by the marching cube grid size), a smooth surface defined from the input boundary samples extracted from volume data. Surface meshes for all segmented objects in Fig. 3 have been generated with our method. These mesh models can then be used for further interactive articulation, deformation, and visualization.

\section{Whole-Body Pregnant Woman Models}

The field of view of the medical images used to extract the UFU include only a part of the maternal body and we chose to use the virtual woman body called Victoria distributed by the software DAZ 3-D Studio (www.daz3d.com) to generate whole-body pregnant woman models.

The Victoria model is easily deformable and can be placed in arbitrary posture, but only represents a body envelope. Recognizing the difficulty to represent the displacement of organs within the maternal body during pregnancy, we do not include them yet. Thus, we simply represent the maternal internal anatomy as a single homogeneous tissue, around the UFU.

\section{A. UFU Insertion}

1) Manual UFU Insertion: In 3-DUS data, the field of view does not include any detailed maternal structures, outside the uterus. The UFU, therefore, needs to be arbitrarily positioned inside Victoria's body. This positioning was performed interactively, using the software Blender (www.blender.org), under the guidance of obstetricians, who could guarantee that the remaining uncertainty in the positioning process was negligible compared to the variability between pregnant women [39]. According to medical experts, the pregnancy starts to be physically visible, around the 13th WG. We, therefore, considered that we did not have to apply any deformation on the model built from 3-DUS images acquired during the first trimester.

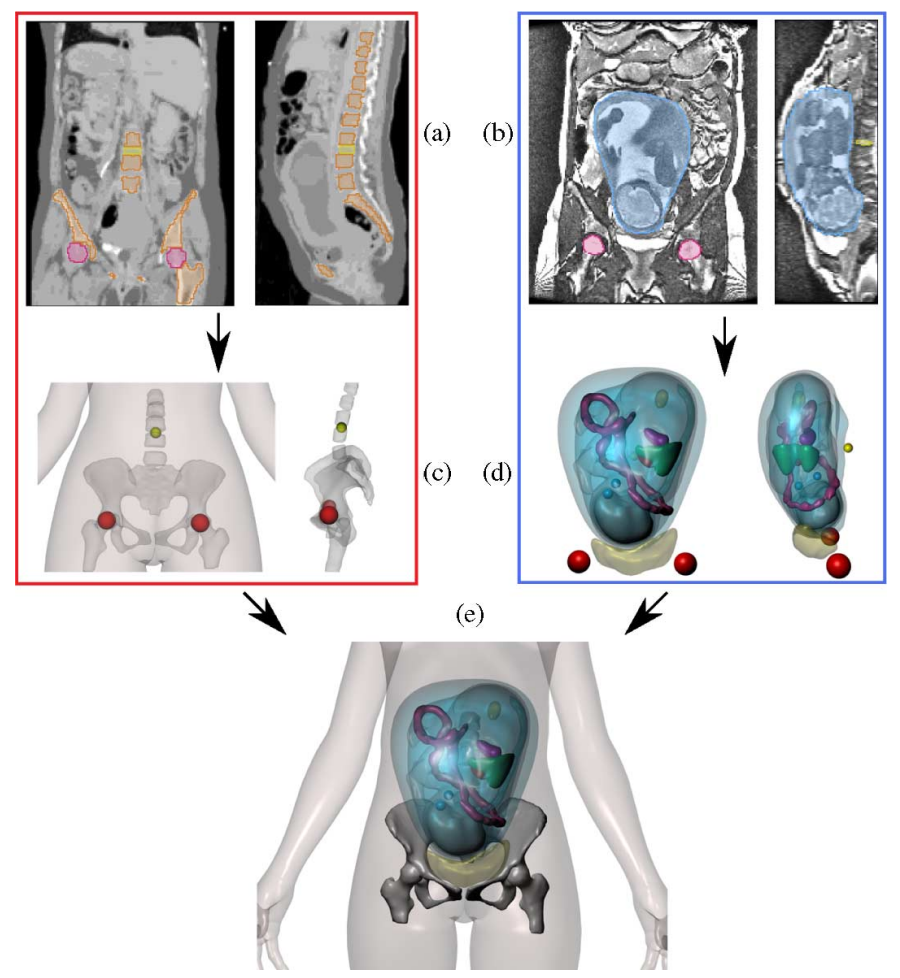

Fig. 6. Insertion of the UFU inside Victoria's body envelope. (a) Segmentation (from a CT dataset) of the skeleton in orange, the femoral heads in red, and the vertebral disk between L3 and L4 in yellow. (b) Segmentation of the uterus in blue, the femoral heads in red, and the vertebral disk between L3 and L4 in yellow from an MRI dataset. (c) Coronal and sagittal views of the reconstructed skeleton and landmark points inserted inside Victoria's body. (d) Coronal and sagittal views of the reconstructed UFU and landmark points. (e) Final positioning of the UFU. In the red frame, the landmark points identification process is performed only once, whereas in the blue frame the process is performed for each new UFU to insert, generating individual models.

When using the UFU models built from MRI segmentation, we need to reshape the nonpregnant body envelope. In the context of a manual insertion, we deform the nonpregnant model Victoria to enlarge her abdominal region and insert the whole UFU inside. All the information and anatomical landmarks available from the limited field of view of the medical images are used. Using Blender, lattice-based FFDs can be defined to fit the maternal envelope visible on the MRI data.

2) Automatic UFU Insertion: Although manual insertion provides satisfactory results, it is time consuming and can vary from one case to another. We, therefore, propose an automated insertion method [40], which creates generic pregnant woman models, not specific to an individual patient. To do so, we needed to add some landmark points inside Victoria's body. We chose to use the pelvic bone, the femoral heads and the lower lumbar vertebrae of a woman, presegmented on a CT dataset of a subject having the same height $(167 \mathrm{~cm})$ as Victoria. These bones and articulation components were meshed and inserted into Victoria's body and three landmarks points were identified: the two femoral head centers and the center of the vertebral disk between the L3 and L4 lumbar vertebrae, as illustrated in Fig. 6.

a) UFU positioning: To automate the UFU positioning inside Victoria, the three landmark points are identified on the 
MRI images used to segment the UFU, and a rigid registration is computed to match these points and the corresponding ones inside Victoria. The rigid transformation (translation and rotation) is computed so as to minimize the distance between the three landmark points selected on the MRI images and the corresponding points in Victoria's partial skeleton, in order to match these points. This transformation is then applied to the whole UFU, in order to register it inside Victoria's body. The registration process proceeds as follows: we first apply a translation to match the center of the segment linking the two femoral heads of the MRI images $h_{i}$ and $h_{i}^{\prime}$ with the ones from Victoria $h_{v}$ and $h_{v}^{\prime}$. We then apply two rotations to align the four landmark points $h_{v}, h_{v}^{\prime}, h_{i}$, and $h_{i}^{\prime}$, and then, make all the landmark points coplanar.

b) Abdomen enlargement: We have chosen the opensource medical simulation framework SOFA [41] to deform the abdomen via physics-based simulations. The SOFA architecture relies on several innovative concepts, in particular, the notion of multimodel representations. The simulation components consist of the woman body envelope, the skeleton, and the UFU, which are defined with three types of representations (visual, behavior, and collision) to optimize rendering, deformation, and collision-detection tasks.

We use the original surface models of the three components for the visual representation and create new models for the behavior and collision representations.

1) For the skeleton, we do not generate behavior nor collision representations, since we only use it as a visual landmark for positioning the UFU.

2) Victoria's body envelope behavior representation is generated from a sparse FFD of the bounding box of the model's trunk. We focus on the deformations of the abdominal wall, considering that the head, arms, and legs do not deform during the simulations. Only the FFD cells containing some matter are considered to generate an hexahedral finite-element model (FEM) of a force field, while the mass is uniformly distributed.

3) The UFU is considered as a rigid object with six degrees of freedom (3-D translation and 3-D rotation). After being registered on the landmarks points, the UFU is scaled down inside Victoria, and then, progressively scaled up back to its original size. For the collision representation between the UFU and Victoria's body, the original models were simplified, via decimation, which strongly improves contact-detection computation.

Finally, all representations are connected to each other using standard mappings. In the following, the segmented uterus will be considered as a rigid structure. Although this is obviously not the case, this assumption allows us to build models, where the position and shape of the uterus match the ones viewed in the acquired images, thus guaranteeing the realism and the fidelity between the images and the proposed models.

c) Contact-detection and collision-response computation: Once the UFU has been rigidly registered inside Victoria's body, the insertion is finalized by simulating the expansion of Victoria's abdomen under the UFU pressure effect. A physicsbased simulation is performed using the image-based collision detection and response method described in [42], which is explained briefly in this section. Before running the simulation, a colliding axis-aligned bounding box (AABB) of each model (i.e., UFU and Victoria) is computed. Then, during the simulations, when the bounding boxes intersect, the intersection surfaces are rendered by the GPU into layered depth images (LDIs), one for each spatial direction. The volume of each object is represented by depth intervals at each pixel, which define an axis-aligned box in 3-D space. They are used to deduce the intersection volume and its gradient. Finally, penalty forces (which try to minimize the intersection volume) are applied to the mesh vertices. These forces are computed as follows:

$$
f=-\lambda \Upsilon \frac{\partial \Upsilon}{\partial x}
$$

where $\lambda$ is an arbitrary positive constraint, $\Upsilon$ is the intersection volume, and $\partial \Upsilon / \partial x$ is the gradient vector. The forces are oriented along the normal of the surface triangles and proportional to their area.

\section{B. Pregnant Woman Morphology}

Since we cannot easily generate patient-based whole-body pregnant women models, we designed a physics-based simulation tool to generate different women morphologies, independent of the UFU content.

1) Fat-Layer Addition: In dosimetry studies, the fat layer plays an important role [43], while its thickness and surface repartition is very variable between women. To add a fat layer to the homogeneous Victoria's body, we have designed an interactive body sculpting tool. The idea is to consider that the body envelope of the generic 3-D woman model is composed of two distinct layers of muscle and fat. The fat layer is initialized from the muscle layer using the morphing tool included in DAZ Studio. Then, its volume can be interactively modified using a mouse pointer. The points of the surface under the pointer are pushed up toward the normal direction by applying a constant force weighted with a Wendland kernel, as in (1). The force applied on the whole surface is propagated with radial basis functions, centered on the 3-D projection of the mouse cursor onto the surface and vanishing within a prescribed Euclidean distance, as expressed in (3).

The surface $\mathcal{S}$ is sampled by the mesh $\mathcal{M}=\{\mathcal{V}, \mathcal{F}\}$ where $\mathcal{V}$ is the set of the vertices and $\mathcal{F}$ is the set of the faces. In our case, the following displacement field is applied on $\mathcal{M}$ :

$$
\forall v_{i} \in \mathcal{V}, f\left(p_{v_{i}}\right)=\alpha \omega\left(q-p_{v_{i}}\right) n_{v_{i}}
$$

where $q$ is the point of the surface $\mathcal{S}$ under the mouse cursor, $p_{v_{i}}$ is the 3-D position of $v_{i}, \omega$ is the Wendland kernel, $n_{v_{i}}$ is the normal of the surface $\mathcal{S}$ at vertex $p_{v_{i}}$ and $\alpha$ is a user-defined weight.

Once the fat layer has been modified, the volume encapsulated between the muscle and fat layers (considered as two independent closed surfaces) is filled with tetrahedra using the isosurface stuffing algorithm [44]. Then, a corotational FEM force field is defined to determine its behavior under gravitational effect. In order to preserve the original fat-layer morphology, points where there is no fat accumulation can also be defined using the mouse. 

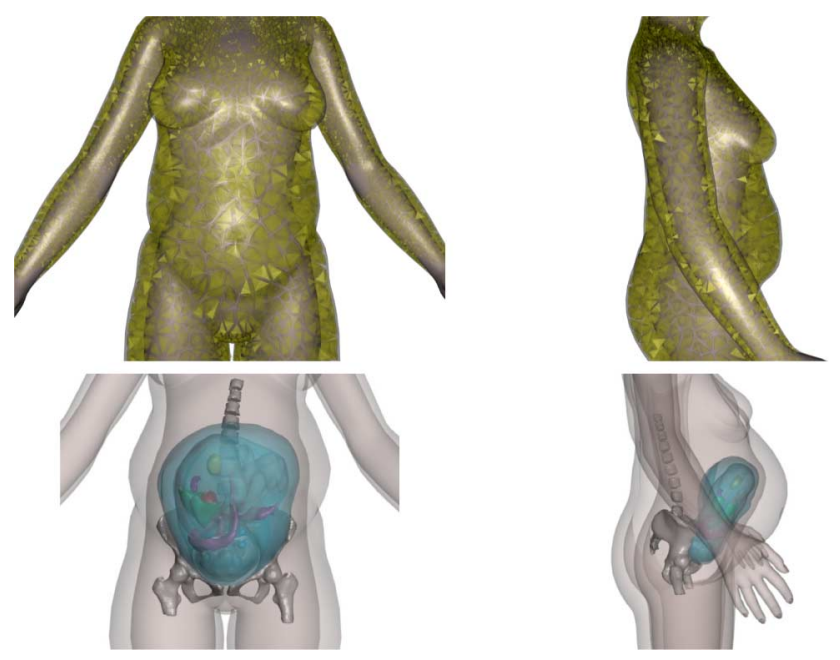

Fig. 7. Coronal and sagittal views of a woman model with an added fat layer represented by yellow tetrahedra, and corresponding whole models, with inserted UFU.

At these point locations, the applied force is always set to zero so that skin and fat layers remain attached, creating a barrier to fat expansion and accumulation. This procedure is necessary to delimit fat "pockets" and prevent unphysiological fat diffusion under gravitational effects.

Fig. 7 illustrates a woman model with an added fat layer.

2) Standing Position Simulation: For dosimetry studies and other applications, we need to generate models in standing positions, while most medical images are acquired with patients in a reclined position, which affects the UFU orientation. To generate a correct standing model, we propose to apply a pendulous movement on the UFU, after insertion inside Victoria's body. This movement is applied via a rotation around the axis defined by the two femoral heads, which enables the UFU to rotate, while pushing the abdominal walls. To simulate the standing position, we tested several rotations of the UFU from $0^{\circ}$ to $50^{\circ}$ between supine and standing positions, and a $20^{\circ}$ rotation was favorably evaluated by obstetricians. However, this parameter can be easily changed in our models, which offer full flexibility and variability (see Fig. 8).

The coarse model created for the collision detection is used to displace the abdominal walls away from the rotating UFU. At the same time, the added fat deforms under the gravitational force field effect, deforming the overall envelope model.

Validation of the whole-body models was performed by clinical collaborators, who could, in particular, estimate the accuracy of the positioning of landmark points and the realism of the reconstruction.

\section{ARticulation of the Fetus}

Articulating the fetus is desirable to change its position inside the maternal uterus and study the impact of the fetal position in dosimetry studies. In order to simulate various fetal positions, we propose to build an armature for the fetus mesh. Similarly

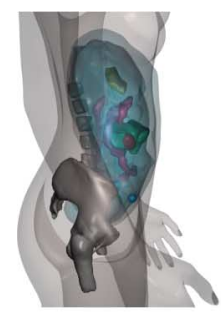

(a)

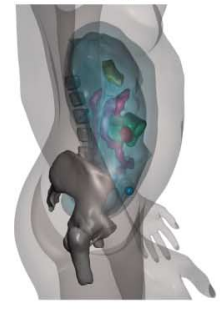

(c)

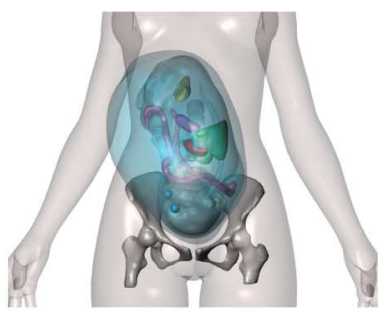

(b)

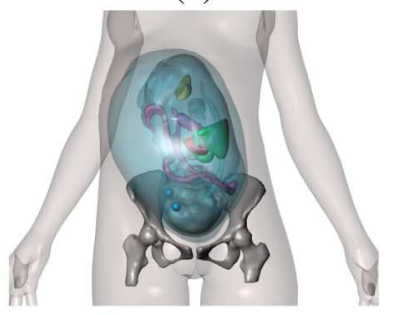

(d)

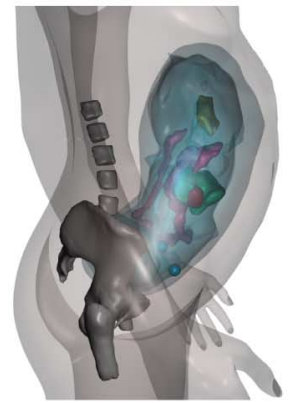

(e)

Fig. 8. (a) and (b) UFU positioning before applying collision-detection methods (sagittal and coronal views). (c) and (d) UFU positioning using collisiondetection methods. (e) Deformed body after the UFU rotation.

to a skeleton, it is composed of pseudobones placed inside the mesh, and associated to each vertex. The articulated fetus was created using the free software Blender.

\section{A. Fetal Bone Armature}

The fetal bone armature is a simplification of the real-fetal skeleton. It is composed of 21 pseudobones, one for each of the following anatomical body parts: head, neck, thorax, abdomen, pelvis, shoulders, arms, forearms, hands, thighs, legs, and feet. To generate individual fetal armatures, we choose to position and scale a generic pseudobone armature, based on the segmentation of the following landmarks on the MRI images: center of the cortex, first cervical vertebra, seventh cervical vertebra, tenth thoracic vertebra, sacrum, urinary bladder, humeral heads, elbows, wrists, metacarpus, femoral heads, knees, ankles, and metatarsus. We exploit 22 landmarks to position and orient the fetal armature inside a segmented fetus mesh.

Table II illustrates the different landmarks and their identifier used to create the armature. Table III lists the pseudobones identifiers of the armature used to represent specific anatomical regions, or body parts, and the landmarks associated with them. The $B_{i}$ pseudobones are actually segments with two landmarks $L_{i}$ as extremities. 
TABLE II

IDENTIFIERS OF THE LANDMARKS USED TO CREATE THE FETAL ARMATURE

\begin{tabular}{|c|c|}
\hline Identifier & Landmarks \\
\hline$L_{1}$ & Cortex center \\
\hline$L_{2}$ & C1 vertebra \\
\hline$L_{3}$ & C7 vertebra \\
\hline$L_{4}$ & T10 vertebra \\
\hline$L_{5}$ & Sacrum \\
\hline$L_{6}$ & Urinary bladder \\
\hline
\end{tabular}

\begin{tabular}{|c|c|}
\hline Identifier & Landmarks \\
\hline$L_{7}, L_{11}$ & Humeral heads \\
\hline$L_{8}, L_{12}$ & Elbows \\
\hline$L_{9}, L_{13}$ & Wrists \\
\hline$L_{10}, L_{14}$ & Metacarpus \\
\hline$L_{15}, L_{19}$ & Femoral heads \\
\hline$L_{16}, L_{20}$ & Knees \\
\hline$L_{17}, L_{21}$ & Ankles \\
\hline$L_{18}, L_{22}$ & Metatarsus \\
\hline
\end{tabular}

When a couple of identifiers is given, the first one is for left body part and the second one for the right part.

TABLE III

IDENTIFIERS OF THE PSEUDOBONES AND THEIR ASSOCIATED LANDMARKS

\begin{tabular}{|c|c|c|}
\hline Identifier & Pseudo-bones & Landmarks \\
\hline$B_{1}$ & Head & $\left(L_{1}, L_{2}\right)$ \\
\hline$B_{2}$ & Neck & $\left(L_{2}, L_{3}\right)$ \\
\hline$B_{3}$ & Thorax & $\left(L_{3}, L_{4}\right)$ \\
\hline$B_{4}$ & Abdomen & $\left(L_{4}, L_{5}\right)$ \\
\hline$B_{5}, B_{9}$ & Shoulders & $\left(L_{3}, L_{7}\right),\left(L_{3}, L_{11}\right)$ \\
\hline$B_{6}, B_{10}$ & Arms & $\left(L_{7}, L_{8}\right),\left(L_{11}, L_{12}\right)$ \\
\hline$B_{7}, B_{11}$ & Forearms & $\left(L_{8}, L_{9}\right),\left(L_{12}, L_{13}\right)$ \\
\hline$B_{8}, B_{12}$ & Hands & $\left(L_{9}, L_{10}\right),\left(L_{13}, L_{14}\right)$ \\
\hline$B_{13}, B_{17}$ & Hips & $\left(L_{5}, L_{15}\right),\left(L_{5}, L_{19}\right)$ \\
\hline$B_{14}, B_{18}$ & Thighs & $\left(L_{15}, L_{16}\right),\left(L_{19}, L_{20}\right)$ \\
\hline$B_{15}, B_{19}$ & Legs & $\left(L_{16}, L_{17}\right),\left(L_{20}, L_{21}\right)$ \\
\hline$B_{16}, B_{20}$ & Foot & $\left(L_{17}, L_{18}\right),\left(L_{21}, L_{22}\right)$ \\
\hline$B_{21}$ & Pelvis & $\left(L_{5}, L_{6}\right)$ \\
\hline
\end{tabular}

When a couple of identifiers is given, the first one is for left body part and the second one for the right part.

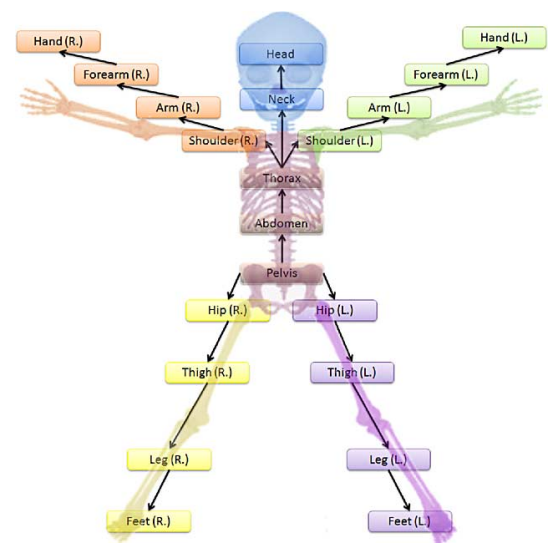

Fig. 9. Pseudobones hierarchical system of the fetal armature with an articulated fetus model overlaid in transparency. The arrows stand for is the parent pseudobone of (R.) stands for right and (L.) for left.

In order to manually change the fetus position, we define a hierarchical ordering of the pseudobones, as illustrated in Fig. 9. For example, the pseudobone representing the pelvis is defined as the parent of the abdominal pseudobone and hip pseudobones; the hip pseudobones are defined as the parents of the thigh pseudobones, etc. This hierarchical ordering defines a pseudobone chain so that when a transformation is applied on a pseudobone (translation, rotation, or scaling), all its children also undergo the same transformation, in a recursive manner.

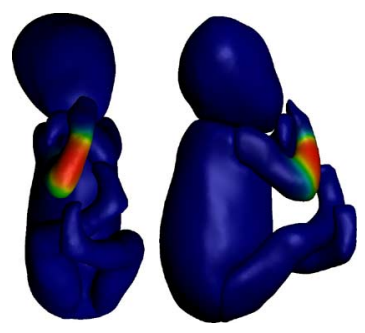

Fig. 10. Vertices weights for the right arm pseudobone generated with the bone heat-weighted method. The weights range from 0 in blue to 1 in red.
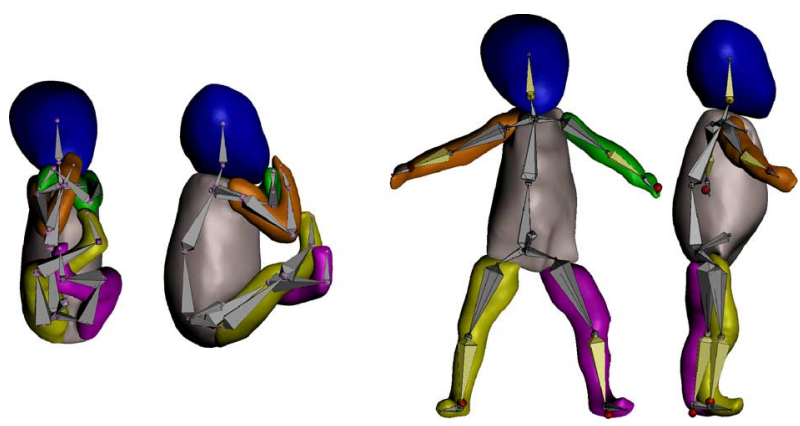

Fig. 11. Front and side view of an articulated fetus model in its original position and in the Vitruvian position.

\section{B. Skinning}

Skinning refers to the association of mesh vertices (in our case, the fetus body envelope) to pseudobones, affecting how vertices follow pseudobones displacements. In practice, each vertex $v_{j}$ is attributed a weight $w_{j}^{i}$ in [0 1 ] for each pseudobone $i$, encoding its sensitivity to pseudobone displacements. The standard linear blend skinning method returns the position $v_{j}^{\prime}$ of the transformed vertex as follows:

$$
v_{j}^{\prime}=\sum_{i} w_{j}^{i} T_{i}\left(v_{j}\right)
$$

where $v_{j}$ is the position of vertex $j, T_{i}$ is the transformation of the $i$ th pseudobone, and $w_{j}^{i}$ is its weight for the vertex $j$.

Skinning was performed with the bone heat-weighted method proposed in [45], where vertex weights are automatically generated based on their proximity to the embedded bones and a spatial smoothing via diffusion over the mesh surface. The diffusion over the surface for the bone $i$ is given by

$$
-\Delta w_{i}+\mathbf{H} w_{i}=\mathbf{H} \mathbf{p}^{i}
$$

where $\Delta$ is the discrete surface Laplacian, calculated with the cotangent formula [46], $\mathbf{p}^{i}$ is a vector with $p_{j}^{i}=1$ if the nearest bone to vertex $j$ is $i$ and $p_{j}^{i}=0$ otherwise, and $\mathbf{H}$ is the diagonal matrix with $H_{j j}$ being the heat contribution weight of the nearest bone to vertex $j$. Fig. 10 illustrates the vertex weights for the right forearm pseudobone of the fetus model.

This skinning method often generates better results than envelope-based methods (where a radius has to be set for each pseudobone to compute the weights), but still suffers from problems caused by linear blend skinning, such as shrinking at flexed joints. Large amplitude transformations of the fetus model can, therefore, induce nonnatural deformations of the envelope. To 


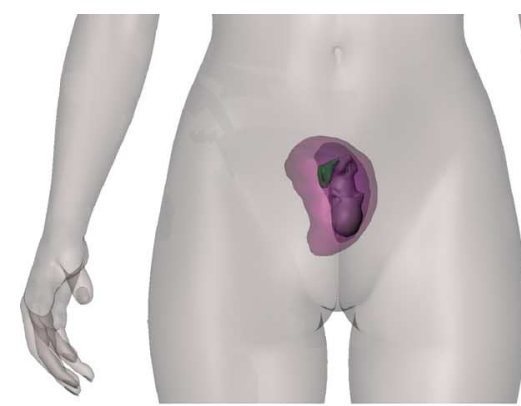

(a)

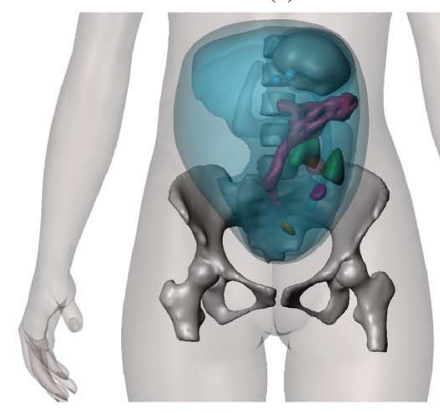

(d)
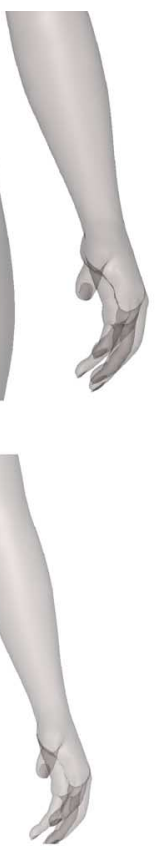
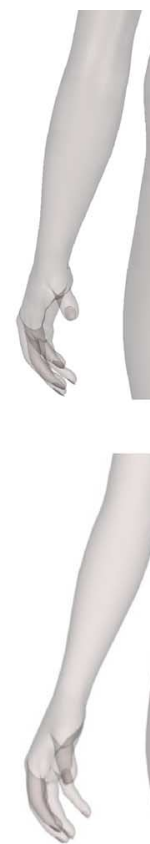

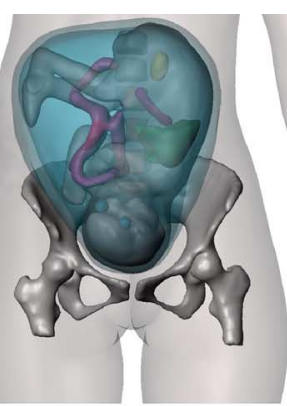

(b)

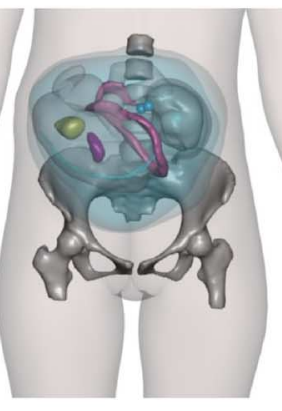

(e)
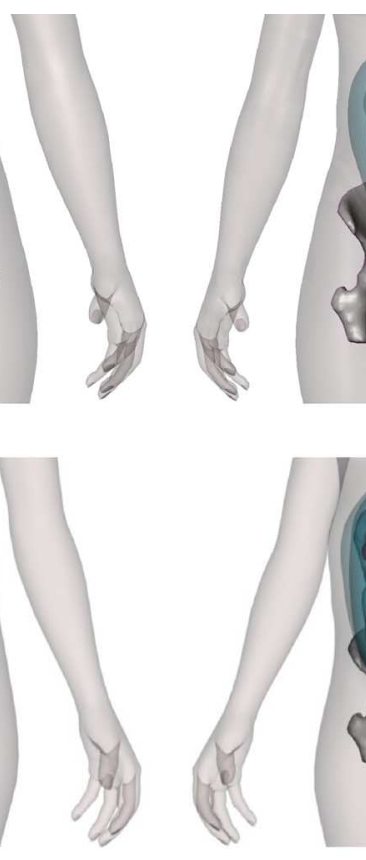

Fig. 12. Coronal views of six different pregnant women with detailed UFU automatically inserted. (a) Fetus at 11 WG built from 3 -DUS data. (b) Fetus in vertex position at $28 \mathrm{WG}$ built from MRI data. (c) Fetus in vertex position at $30 \mathrm{WG}$ with a filled maternal bladder. (d) Fetus in breech position at 31 WG. (e) Fetus in transverse position at $30 \mathrm{WG}$. (f) Twins at $33 \mathrm{WG}$.

avoid such problems, we could use the quaternion deformation interpolation method [47], but still without guarantee of correct final results.

\section{Forward and Inverse Kinematics of the Fetus Model}

To interactively reposition the fetus, we can use a forward kinematics (FK) or inverse kinematics (IK) procedure. With the FK method, only the first bone of the chain (in our case, the pelvis) can be grabbed and moved, since the other bones are attached to their parents. The subsequent bones in the chain can only be rotated or scaled and all the children bones will be rotated or scaled as well. This method is easy to follow, but makes difficult the precise location of the last bones of the chain (in our case hands and feet). With the IK method, the positions of the last bones in the chain are defined, and the other bones reach a position automatically computed by Blender, to preserve the chain without gaps. Precise positioning of hands and feet is therefore much easier with the IK method. Moreover, it allows us to automatically limit the rotation of the limbs. With this method, we are able to interactively and easily define a new position of the fetus that is anatomically correct with the control of our medical experts and test the influence of the fetus position in dosimetry. Fig. 11 shows a fetus placed in the Vitruvian position ${ }^{2}$ using the IK method.

\section{RESUlTS, VALIDATION, AND DisCUSSION}

Sixteen pregnant woman models have been generated at various stages of gestation and in supine/standing positions of the

\footnotetext{
${ }^{2}$ Position of the Vitruvian man drawn by Leonardo da Vinci (1487)
}

mother, using the proposed method. Four were built from 3-DUS images $(6,7,8$, and $11 \mathrm{WG}$ ) and 12 from MRI images (from 24 to $33 \mathrm{WG}$ ). The 3-DUS image datasets enabled us to create the first published pregnant woman models with embryo/fetus models built from medical images, acquired during the first trimester of pregnancy. Models in cephalic, breech, and transverse presentations were built from MRI images, thus covering the main configurations among the large variability of fetal positions [48]. Besides, different implantations of the placenta were also represented, since its location within the uterus varies [49]. Finally, the first pregnant woman model embedding a UFU with twins was built. These different factors of variability are of particular interest, since they have an influence on the distance separating the fetus from the maternal body outer envelop, and hence, on nonionizing dosimetry. Sixteen models are described in details on the web page of the project: http://www.tsi.enst.fr/femonum. All models are made available to the scientific community and can be freely downloaded on this web page for research purpose. Six models are illustrated in Fig. 12.

The models were anatomically validated by our clinical collaborators, who provided routine image data used to precisely measure fetal growth of individual organs. Regarding the segmentation, validation was performed by visual inspection on a number of representative images (for both US and MRI imaging modalities), showing a satisfying accuracy. This type of validation was considered sufficient for the targeted applications, such as nonionizing dosimetry studies, where the main goal is to achieve a good realism of the derived models.

The proposed modeling methodology has enabled us to generate a large set of pregnant women models, but still presents some limitations: segmentation methods are not fully automated, they 
do not include all fetal organs that are visible on MRI, and they require some manual supervision of the meshing procedure. However, the manual interactions needed to overcome these limitations remain reasonable and acceptable for the purpose of this paper. Moreover, the modeling methodology was defined in collaboration with a team of clinician experts, who was able to validate each step.

We have chosen to embed the UFU into a woman body model limited to a body envelope, to propose an automated insertion process, which was approved by the collaborating clinicians. This approach avoids to deform generic models of internal organs, since performing such deformations in a realistic way is a very complex task and still an open issue. We are aware of the limitations of the choice of proposing a homogeneous maternal body model for the accuracy of the dosimetry studies, but this was not the focus of this paper. Our collaborators are currently studying the effect of the homogeneity/heterogeneity of the maternal body on the dosimetry simulations results [50], [51], and this study will probably define the future trend of our work.

Current limitations of the proposed models include: statistical variability of the fetal organs from a set of examples for similar gestational ages need to be studied and encoded in the models, additional organs could be included, especially the fetal skeleton, the major blood vessels, and the placenta in case of MRI data. Discussions with dosimetry experts suggest to work on a more detailed anatomy of the maternal body, especially for the subcutaneous fat layer. Our ongoing work on fat modeling aims at going one step further in the representation of variability of the maternal body morphology.

The set of models, we propose is not representative of the whole population, but each one represents a different fetus in various positions constructed from different pregnant women 3-DUS and MRI scans, which cover different types of configurations, whereas previous works have only focused on single fetus models, eventually scaled to represent several gestational ages. We, therefore, believe that proposing a methodology to generate individual fetus models based on individual imaging data brings some significant value to the field.

\section{CONCLUSION}

In this paper, we have presented a complete methodology to construct hybrid whole-body pregnant woman models with detailed fetal anatomy extracted from 3-DUS and MRI image data. Our approach focuses on the computation of detailed and realistic UFU models, based on imaging data acquired in clinical screening and in the automatization of the modeling process. In this respect, they appear to well complement other existing models.

Methodological developments as well as manual and automated segmentation have enabled us to incorporate in these models several fetal structures, including the brain, the eyes, the heart, the lungs, the urinary bladder, and other tissues, such as the uterus, the amniotic fluid, the umbilical cord, the trophoblast, the myometrium, the endometrium, and the yolk sac depending on the fetus gestational age and the imaging modality.
The proposed modeling method, based on computer graphics tools, allowed us to generate dense and smooth meshes of UFU structures. The placement of the fetus in the synthetic woman and the deformation of the woman abdomen were performed automatically. Segmentation and placement were validated by experienced clinicians.

Preliminary dosimetry studies on models derived from MRI data have been performed and results have shown that fetal position and morphology have a direct influence on fetal exposure [52], demonstrating the importance of considering multiple models with similar gestational ages.

We plan to study in the near future, the influence of the fat thickness for dosimetry simulations. We also plan to refine the woman body model by distinguishing different tissue types, since homogeneous models can lead to an underestimation of the dosimetry.

Our models are not limited to dosimetry studies. They can also be used for medical simulations (such as delivery simulation) or to obtain a good estimation of the fetus weight from the fetal envelope segmentation.

\section{ACKNOWLEDGMENT}

The authors would like to thank the hospitals of St. Vincent de Paul (Prof. C. Adamsbaum), Port-Royal (Prof. G. Grangé), and Beaujon (Prof. D. Luton), Paris, France, for providing the MRI and 3-DUS data and their medical expertise. They would like also to thank Daz 3-D Studio for providing the Victoria model. For more information, illustrations, and download of models for research purpose, please visit our website http://www.tsi.enst.fr/femonum.

\section{REFERENCES}

[1] M. Caon, "Voxel-based computational models of real human anatomy: A review," Radiat. Environ. Biophys., vol. 42, no. 4, pp. 229-235, 2004.

[2] X. Xu and K. Eckerman, Handbook of Anatomical Models for Radiation Dosimetry. New York: Taylor and Francis, 2009.

[3] M. Stabin, E. Watson, M. Cristy, J. Ryman, K. Eckerman, J. Davis, D. Marshall, and M. Gehlen, Mathematical Models and Specific Absorbed Fractions of Photon Energy in the Nonpregnant Adult Females and at the End of Each Trimester of Pregnancy. Springfield, VA: Oak Ridge National Laboratory; National Technical Information Service, 1995.

[4] R. Cech, N. Leitgeb, and M. Pediaditis, "Fetal exposure to low frequency electric and magnetic fields," Phys. Med. Biol., vol. 52, no. 4, pp. 879-888, 2007.

[5] P. J. Dimbylow, "Development of pregnant female, hybrid voxelmathematical models and their application to the dosimetry of applied magnetic and electric fields at 50 hz," Phys. Med. Biol., vol. 51, no. 10, pp. 2383-2394, 2006.

[6] D. Wu, S. Shamsi, J. Chen, and W. Kainz, "Evaluations of specific absorption rate and temperature increase within pregnant female models in magnetic resonance imaging birdcage coils," IEEE Trans. Microw. Theory Tech., vol. 54, no. 12, pp. 4472-4478, Dec. 2006.

[7] X. G. Xu, V. Taranenko, J. Zhang, and C. Shi, "A boundary-representation method for designing whole-body radiation dosimetry models: Pregnant females at the ends of three gestational periods-rpi-p3,-p6 and-p9," Phys. Med. Biol., vol. 52, no. 23, pp. 7023-7044, 2007.

[8] T. Nagaoka, T. Togashi, K. Saito, M. Takahashi, K. Ito, and S. Watanabe, "An anatomically realistic whole-body pregnant-woman model and specific absorption rates for pregnant-woman exposure to electromagnetic plane waves from $10 \mathrm{MHz}$ to $2 \mathrm{GHz}$," Phys. Med. Biol., vol. 52, no. 22, pp. 6731-6743, 2007.

[9] J. Becker, M. Zankl, U. Fill, and C. Hoeschen, "Katja-The 24th week of virtual pregnancy for dosimetric calculations," Pol. J. Med. Phys. Eng., vol. 14, no. 1, pp. 13-19, 2008. 
[10] V. F. Cassola, V. J. de Melo Lima, R. Kramer, and H. J. Khoury, "Fash and mash: female and male adult human phantoms based on polygon mesh surfaces: I. development of the anatomy," Phys. Med. Biol., vol. 55, no. 1, pp. 133-162, Jan. 2010.

[11] M. Cristy and K. F. Eckerman, "Specific absorbed fractions of energy at various ages from internal photon sources," Oak Ridge National Laboratory Report ORNL/TM-8381/V1-V7, 1987, 2010.

[12] C. Shi and X. G. Xu, "Development of a 30-week-pregnant female tomographic model from computed tomography (CT) images for Monte Carlo organ dose calculations," Med. Phys., vol. 31, no. 9, pp. 2491-2497, 2004.

[13] E. Angel, C. Wellnitz, M. Goodsitt, N. Yaghmai, J. DeMarco, C. Cagnon, J. Sayre, D. Cody, D. Stevens, and A. Primak, "Radiation dose to the fetus for pregnant patient undergoing multidetector CT imaging: Monte Carlo simulations estimating fetal dose for a range of gestational age and patient size," Radiology, vol. 249, no. 1, pp. 220-227, 2008.

[14] P. J. Dimbylow, "Induced current densities from low frequency magnetic fields in a $2 \mathrm{~mm}$ resolution, anatomically realistic model of the body," Phys. Med. Biol., vol. 42, no. 3, pp. 221-230, 1998.

[15] J. Chen, "Mathematical models of the embryo and fetus for use in radiological protection," Health Phys., vol. 86, no. 3, pp. 285-295, 2004.

[16] P. J. Dimbylow, "Development of the female voxel phantom, NAOMI, and its application to calculations of induced current densities and electric fields from applied low frequency magnetic and electric fields," Phys. Med. Biol., vol. 50, no. 6, pp. 1047-1070, 2005.

[17] X. G. Xu, T. C. Chao, and A. Bozkurt, "Vip-man: An image-based wholebody adult male model constructed from color photographs of the visible human project for multi-particle monte carlo calculations," Health Phys., vol. 78, no. 5, pp. 476-486, 2000.

[18] T. Nagaoka, S. Watanabe, K. Sakurai, E. Kuneida, M. Taki, and Y. Yamanaka, "Development of realistic high-resolution whole-body voxel models of japanese adult males and females of average height and weight, and application of models to radio-frequency electromagneticfield dosimetry," Phys. Med. Biol., vol. 49, no. 1, pp. 1-15, 2004.

[19] J. Becker, M. Zankl, and N. Petoussi-Henss, "A software tool for modification of human voxel models used for application in radiation protection," Phys. Med. Biol., vol. 52, no. 9, pp. N195-N205, May 2007.

[20] J. Anquez, E. D. Angelini, and I. Bloch, "Segmentation of fetal 3D ultrasound based on statistical prior and deformable model," in Proc. IEEE Int. Symp. Biomed. Imag., 2008, pp. 17-20.

[21] J. Anquez, E. D. Angelini, I. Bloch, V. Merzoug, A. E. BellaicheMillischer, and C. Adamsbaum, "Interest of the steady state free precession (SSFP) sequence for 3D modeling of the whole fetus," in Proc. IEEE Eng. Med. Biol. Soc., 2007, pp. 771-774.

[22] J. Anquez, E. D. Angelini, and I. Bloch, "Automatic segmentation of head structures on fetal MRI," in Proc. IEEE Int. Symp. Biomed. Imag., Boston, MA, Jun. 2009, pp. 109-112.

[23] J. Anquez, L. Bibin, E. D. Angelini, and I. Bloch, "Segmentation of the fetal envelope on ante-natal MRI," in Proc. IEEE Int. Symp. Biomed. Imag., Rotterdam, The Netherlands, Apr. 2010, pp. 896-899.

[24] A. Taflove and S. Hagness, Computational Electrodynamics: The FiniteDifference Time-Domain Method. Norwood, MA: Artech, 2000.

[25] J. Anquez, T. Boubekeur, L. Bibin, E. D. Angelini, and I. Bloch, "Uterofetal unit and pregnant woman modeling using a computer graphics approach for dosimetry studies," in Proc. Med. Image Comput. Comput.Assisted Intervention, London, U.K., Sep. 2009, vol. 5762, pp. 1025-1032.

[26] H. Hoppe, T. DeRose, T. Duchamp, J. McDonald, and W. Stuetzle, "Surface reconstruction from unorganized points," in SIGGRAPH '92: Proc. 19th Ann. Conf. Comput. Graph. Interactive Tech, New York: ACM, 1995, pp. 71-78.

[27] J. L. Bentley, "Multidimensional binary search trees used for associative searching," Commun. ACM, vol. 18, no. 9, pp. 509-517, 1975.

[28] D. Levin, "The approximation power of moving least-squares," Math. Comput., vol. 67, no. 224, pp. 1517-1531, 1998.

[29] D. Levin, "Mesh-independent surface interpolation," in Geometric Modeling for Scientific Visualization, Brunnett, Hamann, and Mueller, Eds., Springer-Verlag, 2003, pp. 37-49.

[30] M. Alexa, M. Gross, M. Pauly, H. Pfister, M. Stamminger, and M. Zwicker, "Point-based computer graphics," in ACM SIGGRAPH Course Notes. New York: ACM, 2004, p. 7.

[31] H. Wendland, "Piecewise polynomial, positive definite and compactly supported radial functions of minimal degree," Adv. Comput. Math., vol. 4, no. 1, pp. 389-396, 1995.

[32] N. Amenta and Y. J. Kil, "Defining point-set surfaces," ACM Trans. Graph., vol. 23, no. 3, pp. 264-270, 2004.
[33] M. Alexa, J. Behr, D. Cohen-Or, S. Fleishman, D. Levin, and C. T. Silva, "Point set surfaces," in Proc. Conf. Vis., 2001, pp. 21-28.

[34] M. Alexa and A. Adamson, "Interpolatory point set surfaces-Convexity and Hermite data," ACM Trans. Graph., vol. 28, no. 2, pp. 1-10, 2009.

[35] D. Bradley, T. Boubekeur, and W. Heidrich, "Accurate multi-view reconstruction using robust binocular stereo and surface meshing," in Proc. Comput. Vis. Pattern Recognit., Jun. 2008, pp. 1-8.

[36] T. Weyrich, M. Pauly, R. Keiser, S. Heinzle, S. Scandella, and M. Gross, "Post-processing of scanned 3D surface data," in Proc. Eurograph. Symp. Point-Based Graph., 2004, pp. 85-94.

[37] L. Kobbelt, M. Botsch, U. Schwanecke, and H.-P. Seidel, "Feature sensitive surface extraction from volume data," in Proc. ACM SIGGRAPH: Proc. 28th Annu. Conf. Comput. Graph. Interactive Tech., 2001, pp. 57-66.

[38] M. Kazhdan, M. Bolitho, and H. Hoppe, "Poisson surface reconstruction," in Proc. 4th Eurograph. Symp. Geometry Process., 2006, pp. 61-70.

[39] L. Bibin, J. Anquez, E. D. Angelini, and I. Bloch, "Hybrid 3D pregnant woman and fetus modeling from medical imaging for dosimetry studies," Int. J. Comput. Assist. Radiol. Surg., vol. 5, no. 1, pp. 49-56, 2009.

[40] J. P. de la Plata Alcalde, L. Bibin, J. Anquez, T. Boubekeur, E. Angelini, and I. Bloch, "Physics-based modeling of the pregnant woman," in Proc. Int. Symp. Biomed. Simul., 2010, pp. 71-81.

[41] J. Allard, S. Cotin, F. Faure, P. Bensoussan, F. Poyer, C. Duriez, H. Delingette, and L. Grisoni, "SOFA-An open source framework for medical simulation," in Proc. Med. Meets Virtual Reality, 2007, pp. 13-18.

[42] F. Faure, S. Barbier, J. Allard, and F. Falipou, "Image-based collision detection and response between arbitrary volumetric objects," in Proc. ACM SIGGRAPH/Eurograph. Symp. Comput. Anim., 2008, pp. 155-162.

[43] J. Wiart, A. Hadjem, N. Gadi, I. Bloch, M. F. Wong, A. Pradier, D. Lautru, V. Hanna, and C. Dale, "Modeling of RF head exposure in children," Bioelectromagnetics, vol. 26, no. S7, pp. S19-S30, 2005.

[44] F. Labelle and J. R. Shewchuk, "Isosurface stuffing: Fast tetrahedral meshes with good dihedral angles," ACM Trans. Graph., vol. 26, no. 3, pp. 1-57, 2007.

[45] I. Baran and J. Popović, "Automatic rigging and animation of $3 \mathrm{~d}$ characters," ACM Trans. Graph., vol. 26, no. 3, pp. 72-1-72-8, 2007.

[46] M. Meyer, M. Desbrun, and P. Schröder, A. H. Barr, "Discrete differentialgeometry operators for triangulated 2-manifolds," in Visualization and Mathematics III. New York: Springer-Verlag, 2003, pp. 35-57.

[47] L. Kavan, S. Collins, J. Zara, and C. O'Sullivan, "Geometric skinning with approximate dual quaternion blending," ACM Trans. Graph., vol. 27, no. 4, pp. 1-23, Oct. 2008, paper 105.

[48] F. Goffinet, M. Carayol, J. Foidart, S. Alexander, S. Uzan, D. Subtil, and G. Bréart, "Is planned vaginal delivery for breech presentation at term still an option? Results of an observational prospective survey in France and Belgium," Amer. J. Obstet. Gynecol., vol. 194, no. 4, pp. 1002-1011, 2006.

[49] L. Kalanithi, J. Illuzzi, V. Nossov, Y. Frisbaek, S. Abdel-Razeq, J. Copel, and E. Norwitz, "Intrauterine growth restriction and placental location," J. Ultrasound Med., vol. 26, no. 11, pp. 1481-1489, 2007.

[50] L. Bibin, J. Anquez, A. Hadjem, E. D. Angelini, J. Wiart, and I. Bloch, "Dosimetry studies on a fetus model combining medical image information and synthetic woman body," Proc. 11th World Congr. Med. Phys. Biomed. Eng., Munich, Germany, Sep. 2009, vol. 25/III, pp. 321-324.

[51] A. Hadjem, E. Conil, J. Anquez, L. Bibin, E. D. Angelini, I. Bloch, and J. Wiart, "Analysis of the SAR induced in the fetus at different stages of gestation exposed to plane wave at $900 \mathrm{MHz}$," presented at the Bioelectromagn. Soc. (BEMS) Annu. Meeting, Seoul, Korea, Jun. 2010.

[52] L. Bibin, J. Anquez, A. Hadjem, E. D. Angelini, J. Wiart, and I. Bloch, "Dosimetry studies on a fetus model combining medical image information and synthetic woman body," presented at the 11th World Congr. Med. Phys. Biomed. Eng., Munich, Germany, 2009.

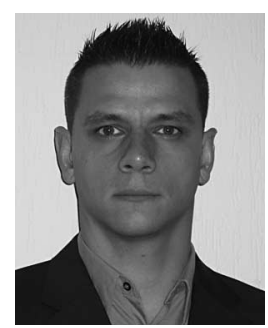

Lazar Bibin received the Ph.D. degree in computer science from the University Paris Descartes, Paris, France, in 2007.

In 2007, he was a Postdoctoral Fellow at Telecom ParisTech, Paris. His current research interests include 3-D medical simulation, medical imaging, computer graphics, image synthesis, and 3-D object modeling. 


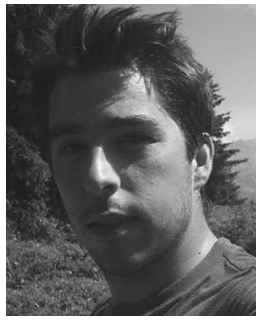

Jérémie Anquez received the B.S. degree in mathematics and informatics from the Paris IX Dauphine University, Paris, France, the M.S. degree in image processing from the Telecom Paris Sud, Evry, France, and the Ph.D. degree from Telecom ParisTech, Paris, in 2009.

$\mathrm{He}$ is currently with Telecom ParisTech. His research interests include image processing (segmentation/registration), anatomical modeling, and ultrasound-guided therapy.

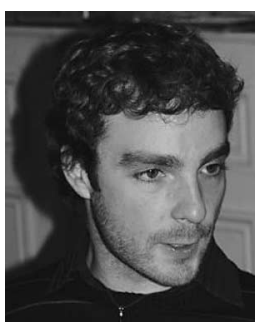

Juan Pablo de la Plata Alcalde received the M.Sc degree in computer science from the Universidad Politecnica, Madrid, Spain, in 2007, and completed the student exchange program ERASMUS from Polytech-Lille (EUDIL), France for one year, in 2005.

He joined the open source framework architecture (SOFA) team in October 2006, within the exchange program LEONARDO, and later became a Full-time Engineer for SOFA. During three years, he participated in the development of an open source framework to simulate any kind of surgical operation. In August 2009, he joined as a Research Engineer the Image Processing and Understanding Group, Telecom Paristech, Paris, where he is engaged in developing a physics-based interactive modeler for pregnant women using the SOFA framework. His research interests include the medical simulation.

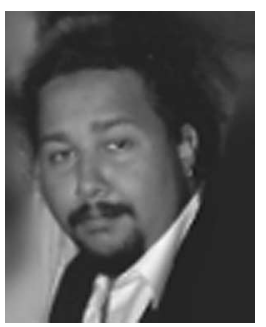

Tamy Boubekeur received the Ph.D. degree from the University of Bordeaux, France, in 2007.

$\mathrm{He}$ is currently an Associate Professor in computer science at Telecom ParisTech (formerly ENST Paris, part of Institut Telecom), the Telecommunication Graduate School, Paris Institute of Technology, Paris, France. From 2004 to 2007, he was a member of the INRIA, Bordeaux, and a Regular Invited Scientist in the Imager Lab, University of British Columbia, Vancouver, Canada. Then, he joined as an Associate Researcher in the Computer Graphics Group, Technical University of Berlin, Germany. In fall 2008, he joined the Department of Signal and Image Processing, Telecom ParisTech, where he was engaged in the computer graphics research activities in the Image Processing and Understanding group. He is an Associate Editor of Computer Graphics Forum (the International Journal of the Eurographics Association). His research interests include 3-D computer graphics, geometric modeling, and real-time rendering.

Dr. Boubekeur was the recipient of several academic awards, including the Günter Enderle Award 2006.

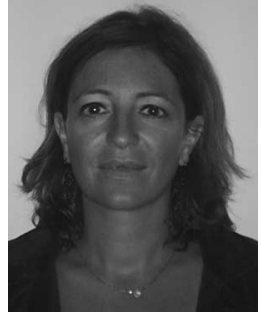

Elsa D. Angelini (M'98) received the B.Sc. degree from Ecole Centrale de Nantes, Nantes, France, in 1996, and the M.Sc. and Ph.D. degrees in biomedical engineering from Columbia University, New York, NY, in 1998 and 2003, respectively.

She is currently an Associate Professor of computer science at the Institut Telecom, Telecom ParisTech, Paris, France. Her research interests include image and signal processing, computer graphics, and scientific visualization for multidimensional biomedical imaging, like denoising, enhancement, segmentation, and modeling.

Dr. Angelini is an Associate Editor for IEEE TRANSACTIONS ON BIOMEDICAL ENGINEERING. She was in the Organizing Committees of IEEE International Symposium Biomedical Imaging 2008 and International Conference on Medical Image Computing and Computer Assisted Intervention 2008. She is a member of the Technical Committee on Medical Imaging and Image Processing of the IEEE Engineering in Medicine and Biology Society.

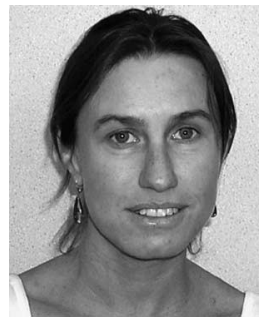

Isabelle Bloch (M'10) graduated from Ecole des Mines de Paris, Paris, France, in 1986, and received the M.Sc. degree from University Paris 12, Paris, in 1987, the Ph.D. degree from Ecole Nationale Supérieure des Télécommunications, Institut Telecom, Telecom ParisTech, Paris, in 1990, and the Habilitation degree from University Paris 5, Paris, in 1995.

She is currently a Professor in the Signal and Image Processing Department and In-charge of the Image Processing and Understanding Group, Télécom ParisTech, Paris, France. Her research interests include 3-D image and object processing, 3-D, fuzzy and logics mathematical morphology, information fusion, fuzzy set theory, structural, graph-based and knowledge-based object recognition, spatial reasoning, and medical imaging. 